\title{
Aptidão física funcional de idosos praticantes de hidroginástica
}

\author{
Functional fitness of elderly practicing aquagym
}

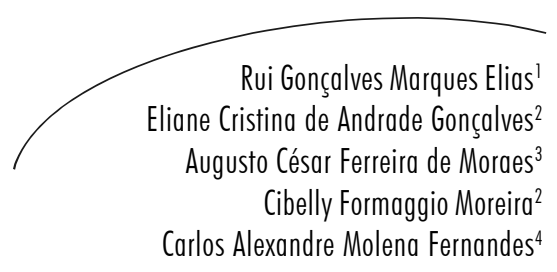

Resumo

O estudo teve como objetivo verificar a aptidão física funcional de idosos praticantes de hidroginástica. A amostra foi constituída por 18 mulheres idosas, com idade média de $65,5 \pm 5,84$ praticantes das aulas de hidroginástica do CEAF (Centro de Excelência em Atividade Física). A aptidão física foi avaliada através da bateria de testes de Rikli e Jones (1999), adaptados por Morrow Jr. (2003), com avaliação de força de membros inferiores, força de membros superiores, flexibilidade dos membros inferiores, mobilidade física (potência, velocidade, agilidade e equilíbrio), flexibilidade dos membros superiores, resistência aeróbia e índice de massa corporal. Os resultados demonstram que os avaliados atingiram bons níveis de aptidão física nos testes de força de membros superior, flexibilidade de membros inferiores e mobilidade física. Já nos testes de força de membro inferior, capacidade aeróbia e flexibilidade de membros superiores, os níveis de aptidão física foram baixos. Sessenta e cinco por cento da amostra apresentaram excesso de peso e obtiveram resultados inferiores na maioria dos testes de aptidão física funcional. Conclui-se que os idosos praticantes de aulas de hidroginástica não apresentaram boa aptidão física funcional geral, principalmente nos níveis de força muscular de membro inferior, desempenho cardiorrespiratório e flexibilidade. Faz-se necessário reavaliar as aulas de hidroginástica, com o objetivo de aumentar gradualmente seu volume e intensidade, para alcançar melhora progressiva das capacidades físicas funcionais da população idosa.

\footnotetext{
Centro de Ciências da Saúde. Universidade Estadual Norte do Paraná-UENP. Jacarezinho, PR, Brasil.

2 Curso de Educação Física. Universidade Estadual de Maringá. Maringá, PR, Brasil.

3 Programa de Pós Graduação em Ciências. Faculdade de Medicina da Universidade de São Paulo. Departamento de Medicina Preventiva. São Paulo, SP, Brasil.

4 Faculdade Estadual de Educação Ciências e Letras de Paranavaí, Departamento de Educação Física. Maringá, PR, Brasil.
}

Palavras-chave: Idosos. Atividade Motora. Saúde do idoso. Aptidão física funcional. Hidroginástica. 


\section{Abstract}

The main objective of this study was to analyze the Functional Fitness of the elderly when practicing aquagym. The study involved the analyses of a group of 18 female individuals with mean age of $65.5 \pm 5.84$, practicing aquagym at the CEAF (Center of Excellence in Physical Activity). The range of tests used in this study was taken from the Rikli and Jones Methods (1999) and adapted by Morrow Jr. (2003), and consisted of analyzing the strengths, flexibilities, agility and balance of the upper and lower limbs, taking into consideration their aerobic resistance and body composition. From the results of these tests we can observe that everyone attained an average or above score during elbow contractions, touching toes whilst seated, standing up and walking, and gained low scores when marching statically for two minutes and bending their arms behind their backs. On the BMI (Body Mass Index) tests, 65\% of the subjects who were overweight or obese scored lower in comparison to the remaining $35 \%$ with normal BMI. Aquagym activities can improve the elderly's functional fitness, especially regarding the flexibility and mobility of upper and lower limbs.
Key words: Elderly. Motor Activity. Health of the Elderly. Functional Fitness. Aquagym.

\section{INTRODUÇÃO}

O envelhecimento é destacado na literatura como uma série de alterações que ocorrem no organismo ao longo da vida. Este processo provoca mudanças nas funções e estrutura do corpo e o torna mais suscetível a uma série de fatores prejudiciais, que podem ser internos ou externos. ${ }^{1-3}$

O impacto do envelhecimento é influenciado pela falta de atividade física, má nutrição, presença de doenças nas fases anteriores da vida e outros fatores, que em geral provocam redução da habilidade motora, do desempenho e do rendimento motor, dificultando a execução das tarefas diárias. ${ }^{4}$

O exercício físico é um importante fator de promoção da saúde, imprescindível para um envelhecimento saudável. Nessa população, se faz necessário dar ênfase aos grandes grupos musculares de natureza rítmica e aeróbica, tais como nadar, andar de bicicleta, caminhadas, hidroginástica, entre outros, que melhore a aptidão física de idosos. ${ }^{5,6}$ Treinamento com pesos em idosos é também uma recomendação do ACMS desde 1998, ${ }^{7}$ pois esta prática pode reduzir a massa corporal, aumentar a massa magra e o conteúdo mineral ósseo, promovendo aumento de força, da massa muscular e da flexibilidade. ${ }^{8,9}$
Programas de exercício físico podem auxiliar idosos a manter sua capacidade funcional, que está diretamente relacionada com a qualidade de vida e independência nesta população. O objetivo da presente pesquisa foi verificar a aptidão física funcional de idosos praticantes de hidroginástica.

\section{METODOLOGIA}

\section{População e amostra}

A amostra foi selecionada de forma intencional, de acordo com os seguintes critérios de inclusão: idade acima de 60 anos, praticantes de hidroginástica no CEAF-UEM. Fizeram parte da amostra 18 idosas com idade média de $64,89 \pm 5,59$ anos.

As aulas foram ministradas sempre pelo período da tarde, numa piscina com profundidade de $1,10 \mathrm{~m}$, medindo $25 \mathrm{~m} \mathrm{x} 12,5 \mathrm{~m}$, com água na temperatura aproximada de 26 a $28^{\circ} \mathrm{C}$. As aulas consistiam em quatro fases: 1 Aquecimento (alongamento e flexibilidade, método estático, durante $5 \mathrm{~min}$ ); 2 - Exercícios aeróbicos (corridas, deslocamentos e movimentos combinados de braços e pernas; 3 - Exercícios localizados (força/resistência dos membros superiores, inferiores e abdominais, utilizando a resistência da água, 4 - Relaxamento (caminhadas lentas, por $5 \mathrm{~min}$ ). 
Instrumentos de medida

O desfecho do presente estudo é a aptidão física funcional. Para as avaliações, utilizou-se a bateria de testes de Aptidão Física Funcional para terceira idade, elaborada por de Rikli \& Jones, ${ }^{10}$ e adaptada por Morrow Jr. et al. ${ }^{11}$ Essa bateria avalia os seguintes domínios: força muscular, resistência muscular, resistência aeróbia, flexibilidade e mobilidade física (agilidade, velocidade e equilíbrio).

A bateria de testes é constituída por oito testes, mas neste estudo foram utilizados apenas sete, por haver dois testes que avaliam a mesma valência física, capacidade aeróbica - o teste de andar seis minutos e o teste de marcha estacionária de dois minutos.

Os testes utilizados neste estudo foram os seguintes: Teste 1 - levantar e sentar na cadeira, avaliação de força e resistência dos membros inferiores; Teste 2 - flexão do antebraço, avaliação da força e resistência dos membros superiores; Teste 3 - estatura e peso, avaliação do IMC (índice de massa corporal); Teste 4 - sentar e alcançar, avaliação da flexibilidade de membros inferiores; Teste 5 - sentado, levantar e caminhar $4,8 \mathrm{~m}$ e voltar a sentar, avaliação da velocidade agilidade e equilíbrio; Teste 6 - alcançar atrás das costas, avaliação da flexibilidade dos membros superiores; Teste 7 - marcha estacionária de dois minutos, avaliação da resistência aeróbia.

\section{Coleta de dados}

Os dados foram coletados no local onde são desenvolvidas as aulas. Foi pedida a permissão dos sujeitos através da assinatura do Termo de Consentimento Livre e Esclarecido, e do coordenador do CEAF-UEM, através da assinatura da Carta de Autorização.

Para a coleta de dados, os testes foram assim divididos: no primeiro dia, foram realizados os testes de levantar e sentar na cadeira e sentar e alcançar; no segundo dia, foram realizados os testes de IMC (peso e altura), alcançar atrás das costas e levantar e caminhar; no terceiro, foram realizados os testes de flexão de cúbito e marcha estacionária de dois minutos.

\section{Análise de dados}

Os dados foram analisados por meio de estatística descritiva, utilizando média, desvio padrão e percentil. Para melhor compreensão dos resultados dos percentis, foram interpretados $\mathrm{da}$ seguinte forma: percentil 10, 25 e 50 - baixa aptidão; e percentil 75 e 90 - boa aptidão. Em relação ao índice de massa corpórea (IMC), foi utilizada a classificação de acordo com a Organização Mundial da Saúde, ${ }^{12}$ sendo que neste estudo foi criada a categoria "Excesso de Peso", que é a junção da classificação sobrepeso (IMC $=25$ a $29,9 \mathrm{~kg} / \mathrm{m}^{2}$ ) e obesidade (IMCe" 30 $\left.\mathrm{kg} / \mathrm{m}^{2}\right)$. O teste qui-quadrado foi utilizado para verificar associação índice de massa corpórea (IMC) dos avaliados com os resultados dos testes de aptidão física.

\section{Procedimentos Éticos}

O projeto foi aprovado pelo Comitê de Ética Permanente de Pesquisa da Universidade Estadual de Maringá, e aprovado sob o parecer $n^{\circ} 500 / 09$. Todos os participantes assinaram o termo de consentimento livre e esclarecido.

\section{RESULTADOS}

Os resultados demonstram, segundo a tabela 1 , que nos testes de flexibilidade dos membros superiores, flexibilidade de membros inferiores e mobilidade física, obtiveram-se uma elevada incidência de idosas classificadas com baixa aptidão física. 
Tabela 1 - Resultados dos testes de aptidão física de idosos praticantes de hidroginástica, Maringá-Pr, $2009(n=18)$.

\begin{tabular}{lccccc}
\hline \multicolumn{1}{c}{ Teste } & Baixa Aptidão & \multicolumn{2}{c}{ Boa } \\
Aptidão
\end{tabular}

A tabela 2 apresenta o índice de massa corporal da amostra de acordo com a classificação da Organização Mundial da
Saúde. ${ }^{12}$ Verificou-se alta prevalência de excesso de peso $(61,11 \%)$ entre as idosas praticantes de hidroginástica.

Tabela 2 - Índice de Massa Corporal de idosos praticantes de hidroginástica. Maringá-Pr, 2009 (n=18).

\begin{tabular}{lcc}
\hline Indice de Massa Corporal & F & $\%$ \\
\hline Baixo peso & 0 & 0 \\
Normal & 7 & 38,89 \\
Excesso de peso & 11 & 61,11 \\
\hline
\end{tabular}

$\mathrm{Na}$ associação entre o índice de massa corpórea (IMC) dos avaliados com os resultados dos testes de aptidão física (tabela 3), foi verificada associação entre excesso de peso e baixo nível de aptidão física nos testes de mobilidade física de membros inferiores, resistência aeróbia e flexibilidade de membros superiores. 
Tabela 3 - Relação entre índice de massa corporal e testes de aptidão física de idosos praticantes de hidroginástica. Maringá-Pr, $2009(\mathrm{n}=18)$.

\begin{tabular}{|c|c|c|c|}
\hline TESTE / IMC & $\begin{array}{c}\text { Peso } \\
\text { Normal }\end{array}$ & Excesso de Peso & $\mathrm{P}$ \\
\hline
\end{tabular}

Flexibilidade de membros superiores

$\begin{array}{clllll}\text { Baixa Aptidão } & 4 & 57,14 & 6 & 33,33 & 0,9139 \\ \text { Boa Aptidão } & 3 & 42,86 & 5 & 27,78 & \end{array}$

Força de membros superior es

$\begin{array}{crrrrr}\text { Baixa Aptidão } & 2 & 28,57 & 10 & 55,56 & 0,0062 \\ \text { Boa Aptidão } & 5 & 71,43 & 1 & 5,56 & \end{array}$

Flexibilidade de membros inferiores

$\begin{array}{clllll}\text { Baixa Aptidão } & 4 & 57,14 & 2 & 11,11 & 0,8740 \\ \text { Boa Aptidão } & 3 & 42,86 & 9 & 50,00 & \end{array}$

Força de membros inferiores

$\begin{array}{clllll}\text { Baixa Aptidão } & 5 & 71,43 & 8 & 44,44 & 0,9522 \\ \text { Boa Aptidão } & 2 & 28,57 & 3 & 16,67 & \end{array}$

Mobilidade física

$\begin{array}{crrrrr}\text { Baixa Aptidão } & 5 & 71,43 & 11 & 61,11 & 0,0601 \\ \text { Boa Aptidão } & 2 & 28,57 & 0 & 0,00 & \end{array}$

Resistência aeróbia

\begin{tabular}{crrrrr} 
Baixa Aptidão & 4 & 57,14 & 10 & 55,56 & 0,0930 \\
Boa Aptidão & 3 & 42,86 & 1 & 5,56 & \\
\hline
\end{tabular}

\section{DISCUSSÃO}

São descritos atualmente vários testes para a mensuração da aptidão física no adulto idoso. Neste foi utilizado o de Rikli \& Jones, ${ }^{10}$ por ser mais completo, prático, replicável e de baixo custo operacional. Outra vantagem é que se trata de um teste já validado. ${ }^{13}$

Os níveis de aptidão da força dos membros inferiores encontrado foram baixos. Uma possível explicação é que idosos apresentam diminuição $\mathrm{da}$ força muscular, principalmente nos membros inferiores. ${ }^{14}$ Os baixos níveis de atividade física (sobretudo locomoção ativa) estão associados à menor força muscular dos membros inferiores. Este fato acontece pela menor utilização da musculatura com o passar dos anos, principalmente dos membros inferiores, uma vez que os idosos diminuem a atividade física, permanecendo a maior parte do tempo sentados, com baixa locomoção. ${ }^{15}$ Sugere-se, portanto, que sejam colocadas cargas (pesos) nos membros inferiores durante a realização das aulas de hidroginástica para aumentar a força desses membros.

Foi encontrado na literatura um aumento significativo na força dos membros superiores quando avaliados através do teste de flexão de cúbito após 12 e 19 semanas de treinamento de força com pesos livres em idosas. Os resultados encontrados no presente estudo se assemelham com pesquisas que utilizaram exercícios de força específicos, podendo comparar então que, na população idosa, a hidroginástica tem efeitos semelhantes a exercícios de resistência de força muscular, muitas vezes realizado em academias. ${ }^{16-18}$ 
Em pesquisa realizada com idosos que praticavam caminhadas e outras atividades físicas, verificou boa aptidão no teste de resistência aeróbia..$^{19}$ Já neste estudo, as idosas praticantes de hidroginástica obtiveram baixo desempenho no teste de resistência aeróbia, sugerindo que a hidroginástica pouco contribui para o desempenho cárdio-respiratório.

A mobilidade física parece estar relacionada com o tempo de prática de atividade física em idosos. Em estudo longitudinal de dois anos de exercício físico para idosas, não se verificou melhora estatística na mobilidade física. No entanto, em um acompanhamento de 12 anos, o programa de exercícios físicos melhorou significativamente esta capacidade física. ${ }^{20-22}$

No teste de flexibilidade, os resultados encontrados são semelhantes aos achados na literatura, ${ }^{23}$ que também obtiveram resultados positivos em relação à flexibilidade em estudo com idosas que participaram de um programa de atividades de flexibilidade e alongamento por 24 semanas.

Quando analisado o IMC deste grupo, foi encontrada alta prevalência de sobrepeso e obesidade, resultado que pode estar relacionado a vários fatores como, por exemplo, má alimentação, frequência nas aulas de hidroginástica, ou ainda tipo de treinamento aplicado nas aulas, intensidade e volume da mesma. ${ }^{24}$

O ganho no peso corporal e o acúmulo da gordura corporal no idoso parecem resultar de um padrão programado geneticamente, de mudanças na dieta e no nível de atividade física, relacionados a uma interação entre esses fatores. ${ }^{25}$ Em contrapartida, estudo com idosas submetidas a oito meses de treinamento com hidroginástica verificou diminuição da massa corporal e nos perímetros de cintura, glúteo, coxa e panturrilha. ${ }^{26}$

No presente estudo, verificou-se associação entre o excesso de peso e baixa aptidão física nos testes de força de membros superiores $(\mathrm{p}=0,062)$. Estes resultados são compatíveis com outro estudo que verificou, em sua pesquisa com idosas praticantes de hidroginástica e natação, que a maioria das idosas com IMC acima do normal apresentou um índice de aptidão funcional geral (IAFG) baixo, enquanto a maioria das idosas com IMC normal apresentou um IAFG bom. ${ }^{27} \mathrm{Da}$ mesma forma, um estudo com 1.451 idosos verificou que a obesidade está inversamente relacionada à aptidão física dos idosos. ${ }^{28,29}$

Sendo assim, sugere-se que os treinos de hidroginástica sejam reavaliados, para que haja diminuição do peso corporal, pois foi detectada relação direta entre índice de massa corporal e aptidão física.

\section{CONCLUSÃO}

Conclui-se que as idosas praticantes de aulas de hidroginástica não apresentaram boa aptidão física funcional geral, sobretudo nos níveis de força muscular de membro inferior, desempenho cardiorrespiratório e flexibilidade.

Observou-se, por fim, que para melhor da aptidão física funcional e reduzir o peso corporal, é necessário reavaliar as aulas de hidroginástica, com o objetivo de aumentar gradualmente o volume e intensidade dessas, alcançando assim uma melhora progressiva das capacidades físicas funcionais da população idosa. 


\section{REFERÊNCIAS}

1. Santos $\mathrm{K}$, et al. Fatores associados com a incapacidade funcional em idosos do Município de Guatambu. Cad Saúde Pública 2007 nov; 23(11): 2781-8.

2. Siqueira RL, Botelho MIV, Coelho FMG. A velhice: algumas considerações teóricas e conceituais. Ciênc Saúde Colet 2002; 7(4): 899 906.

3. Padilha AL, Delgado EIA. Melhor idade do Brasil: aspectos biopsicossociais decorrentes do processo de envelhecimento. Ulbra e Movimfum 2010; 1(2) :76-91.

4. Albala C, et al. Encuesta Salud, Bienestar y Envejecimiento (SABE): metodología de la encuesta y perfil de la población estudada . Rev Panam Salud Pública 2005;17(5-6):307-322.

5. Britto RR, Santiago L, Elisa P, Pereira LSM. Efeitos de um programa de treinamento físico sobre a capacidade funcional de idosos institucionalizados. Tex Envelhec 2005; 8(1): 9-20.

6. Ferreira L, Barbosa TD, Gobbi S, Arantes LM. Capacidade funcional em mulheres jovens e idosas: projeções para uma adequada prescrição de exercícios físicos. Rev Educ Fís 2008;19(3): 403-412.

7. American College of sports Medicine (ACSM). Position Stand on The recommended quantity and quality of exercise for developing and maintaining cardiorespiratory and muscular fitness, and flexibility in adults. Med Sci Sports Exerc 1998; 30:975-91.

8. Santos CF, et al. Avaliação da composição corporal por DEXA em homens com idade superior a 60 anos submetidos a um programa de treinamento com pesos. Rev Bras Med 2010; 67: 141-146.

9. Hunter GR, McCarthy JP, Bamman MM. Effects of resistance training on older adults. Sports Med 2004: 34(5):329-348.

10. Rikli RE, Jones CJ. Development and validation of a functional fitness test for communityresiding older adults. J Aging Phys Activity 1999; 7(2): 129-161.

11. Morrow Jr JR. Medida e avaliação do desempenho humano. 2.ed. Porto Alegre: Artmed; 2003.

12. Word Health Organization (WHO). Obesity: preventing and managing the global epidemic. Geneva: WHO; 1997.

13. Rikli RE, Jones CJ. Reliability, validility, and methodological issues in assessing physical activity in older adults. Res Q Exerc Sport 2000; 71(4): 89-96.

14. Matsudo SMM, Matsudo VKR, Barros Neto TL. Evolução do perfil neuromotor e capacidade funcional de mulheres fisicamente ativas de acordo com a idade cronológica. Rev Bras Med Esporte 2003; 9(6): 365-376.

15. Krause MP, Buzzachera CF, Hallage T, Pulner $\mathrm{SB}$, Silva SG da. Influência do nível de atividade física sobre a aptidão cardiorrespiratória em mulheres idosas. Rev Bras Med Esporte 2007; 13(2) 97-102

16. Kura GG, Ribeiro LSP, Niquetti R, Filho HT. Nível de atividade física, IMC e índices de força muscular estática entre idosas praticantes de hidroginástica e ginástica. Rev Bras Ciên Envelhecimento Hum 2003; 7(2):30-40.

17. Buzzachera CF, et al. Efeitos do treinamento de força com pesos livres sobre os componentes da aptidão funcional em mulheres idosas. Rev Educ Fís. 2008; 19(2):195-203.

18. Teixeira DC, Prado Junior SRR, Lima DF, Gomes SC, Brunetto AF. Efeitos de um programa de exercício físico para idosas sobre variáveis neuromotoras, antropométrica e medo de cair. Rev. Bras. Educ Fís Esp. 2007; 21(2):107120.

19. Gonçalves R, Gurjão ALD, Gobbi S. Efeitos de oito semanas do treinamento de força na flexibilidade de idosos. Rev Bras Cineantropom Desempenho Hum 2007; 9(2):145-153.

20. Júnior JPS, et al. Estabilidade das variáveis de aptidão física e capacidade funcional de mulheres fisicamente ativas de 50 a 89 anos. Rev Bras. Cineantropom Desempenho Hum 2011; 13 (1):8-14.

21. Pauli JR, Souza LS, Zago AS, Gobbi S. influência de 12 anos de prática de atividade física regular em programa supervisionado para idosos. Rev Bras Cineantropom Desempenho Hum 2009; 11(3):255-60.

22. Mendonça TT, Ito RE, Bartholomeu T, Tinucci Forjaz CLM. Risco cardiovascular, aptidão física e prática de atividade física de idosos de um parque de São Paulo. Rev Bras Ciênc Movim 2004; 12(2):19-24.

23. Varejão RV, Dantas EHM, Matsudo SMM. Comparação dos efeitos do alongamento e do flexionamento, ambos passivos, sobre os níveis de flexibilidade, capacidade funcional e qualidade de vida do idoso. Rev Bras Ciênc Movim 2007; 15(2):87-95. 
24. Valle RGR. Programa de atividade física para terceira idade e sua relação com as medidas antropométricas e aptidão física. Rev Bras Obes Nut Emagrecimento 2007; 1(4):71-76.

25. Matsudo SM, Matsudo VKR, Barros Neto TL. Impacto do envelhecimento nas variáveis antropométricas, neuromotoras e metabólicas da aptidão física. Rev Bras Ciênc Movim 2000; 8(4):21-32.

26. Gubiane GL, Neto CSP, Petroski EL, Lopes AS. Efeitos da hidroginástica sobre indicadores antropométricos de mulheres entre 60 e 80 anos de idade. Rev Bras Cineantropom Desempenho Hum 2001; 3(1):34-41.
27. Mazo GZ, Külkamp W, Lyra VB, Prado APM. Aptidão funcional geral e índice de massa corporal de idosas praticantes de atividade física. Rev Bras Cineantropom Desempenho Hum 2006; 8(4): 46-51.

28. Barreto SM, Passos VMA, Lima-Costa MFF. Obesidade e baixo peso entre idosos brasileiros: projeto Bambuí. Cad Saúde Pública 2003; 19(2): 605-612.

29. Melo GF, Giavoni A. Comparação dos efeitos da ginástica aeróbica e da hidroginástica na composição corporal de mulheres idosas. Rev Bras Ciênc Movim. 2004; 2(2): 13-18. 